
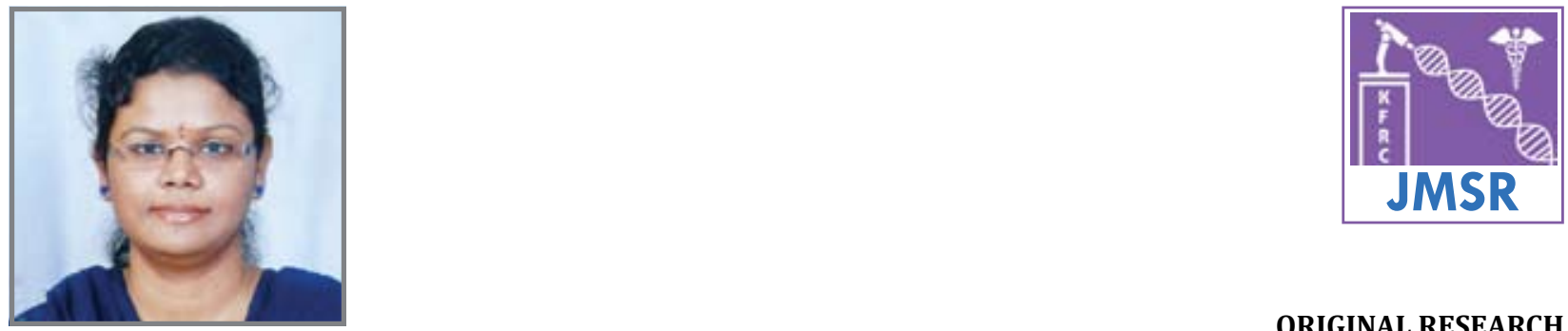

Dr. Madhavi Latha Bommala

ORIGINAL RESEARCH

\title{
Detection of antinuclear antibodies by indirect immunofluorescence method and its comparison with line immunoassay in a tertiary care hospital: A laboratory based observational study
}

Dr. Madhavi Latha Bommala ${ }^{1, *}$ and Dr. Anil Kumar Bilolikar ${ }^{1}$

${ }^{1}$ Department of Microbiology, Krishna Institute of Medical Sciences, Minister Road, Secunderabad-500003, Telangana, India

\begin{abstract}
Antinuclear antibodies (ANA) are the hallmark of autoantibody production in autoimmune diseases and its testing is widely used as screening test in autoimmune diseases. ANA are directed against components of the cell nuclei such as DNA, histones, nucleoli and ribonucleoprotein. ANA are detected by indirect immunofluorescence (IIF) assay, which is among the most commonly used routine method for ANA detection as screening test, due to its ability to detect multiple antigens simultaneously. In this study, serum samples, referred to our laboratory for ANA testing were subjected for testing by IIF method and line immunoassay (LIA) during a study period of 20 months and the two were correlated with one another to establish any link between the two. A total of 279 serum samples were processed for ANA testing during the study period from June 2012 to January 2014. Of these 279 samples, $199(71.3 \%)$ were ANA IIF positive and $80(28.7 \%)$ were ANA IIF negative. The spectrum of various positive ANA IIF patterns are nucleus homogenous 52(26.1\%), nucleus granular 50(25.1\%), mixed pattern $57(28.6 \%)$, mitosis positive $15(7.5 \%)$, nucleus nucleolar $13(6.5 \%)$, nucleus dotted $8(4 \%)$, nuclear membrane $1(0.5 \%)$, cytoplasm positive $3(1.5 \%)$. All the samples tested by ANA IIF were subjected to LIA. Of these $159(56.9 \%)$ were both ANA IIF and LIA positive. In addition, $40(14.3 \%)$ samples were detected as IIF positive but LIA negative, whereas the rest $14(5 \%)$ samples were IIF negative but LIA positive. In the present study, a definite correlation was found in 201(71\%) samples between ANA patterns and the LIA. Thus ANA IIF method using biochips can be used as a cost effective screening method for ANA testing and restricting LIA, which are expensive. This could economize on the cost of laboratory investigations in a developing country like India.
\end{abstract}

Keywords: Antinuclear antibodies; Indirect immunofluorescence method; Immunoassay; Autoimmune diseases; Tertiary care hospital; Observational study

*Corresponding author: Dr. Madhavi Latha Bommala, Department of Microbiology, Krishna Institute of Medical Sciences, Minister Road, Secunderabad-500003, Telangana, India. Email: madhavilatha.bommala@gmail.com

Received 12 June 2014; Revised 25 August 2014; Accepted 03 September 2014

Citation: Madhavi Latha B, Anil Kumar B (2014) Detection of antinuclear antibodies by indirect immunofluorescence method and its comparison with line immunoassay in a tertiary care hospital: A laboratory based observational study. J Med Sci Res 2(4):194-199. DOI: http://dx.doi.org/10.17727/JMSR.2014/2034

Copyright: (C) 2014 Madhavi Latha B, et al. This is an open-access article distributed under the terms of the Creative Commons Attribution License, which permits unrestricted use, distribution, and reproduction in any medium, provided the original author and source are credited. 


\section{Introduction}

Autoimmune diseases are conditions in which the immune system damages specific organs or tissues and are characterized by the presence of antinuclear antibodies (ANA) in the blood of the patients. ANA are specific antibodies directed against a variety of nuclear antigens which have the capability of binding and destroying certain structures within the nucleus of the cells and are detected in the serum of the patients with rheumatic \& non rheumatic diseases [1]. Although lower amounts of these antibodies are seen in normal individuals, an increase in titres are exclusively seen in patients with autoimmune diseases and serve as markers for diagnosis and prognosis [2]. These antibodies are involved not only in disease pathogenesis but also constitute basis for diagnosis and treatment [1]. Their detection with high sensitivity and specificity is therefore of utmost importance. The first description of ANA test began with the seminal discovery of the lupus erythematosus (LE) cell and its phenomenon by Hargraves (1949) [3]. ANA testing is widely used as a screening test in connective tissue diseases such as systemic lupus erythematosus, rheumatoid arthritis, systemic sclerosis, sjogren syndrome, juvenile idiopathic arthritis, polymyositis, dermatomyositis, scleroderma, CREST (calcinosis, Raynaud's phenomenon, esophageal motility abnormalities, sclerodactyly and telangiectasia) syndrome and mixed connective tissue disorders.

Various detection methods are in use and there is continuous outpouring of newer techniques like flow cytometry and nanotechnology for the diagnosis and therapeutic monitoring [4]. Indirect immunofluorescence test (IIF) on Hep-2 (human epithelial cell tumour line) is a classical technique for detection of ANA and is considered as "gold standard" for testing for ANA in clinical practice with high sensitivity [5]. Though positive fluorescence staining indicates the presence of ANA, it does not however allow precise identification of these antibodies. For this specialized techniques like ELISA, western blotting or line immunoassay (LIA) are employed [6].

The primary objective of this study is i) To know the seroprevalance of ANA in the study population, ii) Detection of various ANA patterns by IIF method, iii) Comparison of ANA IIF with LIA and to find if a definite correlation exists between the two methods, so that IIF could be used as the routine screening test while restricting the use of LIA which is expensive.

\section{Materials and methods}

The present study was conducted in the department of Microbiology, KIMS Hospital, during the study period of 20 months from June 2012 to January 2014. It is a laboratory based observational study. Clinical correlation was not done.

Serum samples of patients who sought medical help for rheumatic disease/ non-rheumatic disease by rheumatologists/ neurologists/ nephrologists/ physicians/ dermatologists or from any other hospital departments for diagnosis of autoimmune diseases were processed for ANA testing by indirect immunofluorescence (IIF) and line immunoassay.

Only samples received for both IIF and LIA testing are included. Samples received for single test were excluded from the study. Fresh whole blood sample were collected in red or yellow vacutainers and serum was separated from the clotted blood samples by centrifugation as per standard protocol.

\section{Test procedure- ANA IIF \& LIA}

The patientserum samples were processed in dilution 1:100 with PBS (Phosphate buffer solution) using Hep-2010/ liver biochip (Monkey) [EUROIMMUNE AG]. The test procedure was carried out as per the kit insert. Positive and negative controls were run with each test daily.

The BIOCHIP slide was observed under fluorescent microscope at $\mathrm{x} 400$ and based on the intensity of positive and negative controls, the fluorescent intensity of the samples were graded and a score of $1+$ to $4+$ was given. The test result was discarded if the positive control sample failed to show precise results. The serum samples which were positive or negative by IIF method were further processed by Line immunoassay.

Nylon strips coated with 15 highly purified antigens as discrete lines with plastic backing [EUROIMMUNE AG] coated with antigens nRNP/ Sm, Sm, SSA, 
Ro-52, SSB, Scl-70, PM-Scl, Jo-1, CENP-B, dsDNA, Nucleosomes, Histones, Ribosomal P-protein, AMAM2 were used along with control band. The patient serum samples were processed in dilution 1:100 with PBS (Phosphate buffer solution). The test procedure was carried out as per the kit insert.

Then the strips were dried and evaluated by comparing the intensity of the reaction with positive control line by image analysis.

\section{Results}

In this study, out of 279 samples, 236 (84.5\%) were from female patients and 43(15.5\%) were from male patients (Table 1). Out of 236 female patient samples, ANA IIF was positive in $171(72.4 \%)$ and negative in $65(27.6 \%)$. Out of 43 male patient samples, ANA IIF was positive in $28(65.2 \%)$ and negative in $15(34.8 \%)$. Whereas ANA LIA was positive in $147(62.2 \%)$ and negative in $89(37.8 \%)$ female patient samples. Out of 43 male patient samples, ANA LIA was positive in 23(53.4\%) and negative in 20(34.8\%).

Table 1: Gender wise distribution of ANA.

\begin{tabular}{|lcllll|}
\hline \multirow{2}{*}{ Gender } & Total number of & \multicolumn{2}{c|}{ Samples processed by ANA IIF } & \multicolumn{2}{c|}{ Samples processed by ANA LIA } \\
\cline { 3 - 6 } & samples & Positive & Negative & Positive & Negative \\
\hline Female & $236(84.5 \%)$ & $171(72.4 \%)$ & $65(27.6 \%)$ & $147(62.2 \%)$ & $89(37.8 \%)$ \\
Male & $43(15.5 \%)$ & $28(65.2 \%)$ & $15(34.8 \%)$ & $23(53.4 \%)$ & $20(34.8 \%)$ \\
\hline
\end{tabular}

In the present study most of the ANA positives were 30 years and 51-60 years age group (Table 2). seen in 41-50 years and 31-40 years followed by 21-

Table 2: Age Distribution of ANA IIF.

\begin{tabular}{|cccc|}
\hline $\begin{array}{c}\text { Age group } \\
\text { (in years) }\end{array}$ & Total number of samples & $\begin{array}{c}\text { Number of samples positive } \\
\text { by ANA IIF }\end{array}$ & $\begin{array}{c}\text { Number of samples } \\
\text { negative by ANA IIF }\end{array}$ \\
\hline $0-10$ & 2 & 2 & 0 \\
$11-20$ & 15 & 14 & 1 \\
$21-30$ & 48 & 37 & 23 \\
$31-40$ & 76 & 53 & 24 \\
$41-50$ & 78 & 54 & 14 \\
$51-60$ & 38 & 24 & 7 \\
\hline
\end{tabular}

Among the 279 ANA samples processed, 199(71.3\%) samples were ANA IIF positive in 1:100 serum dilutions. Of these positive ANA IIF, 159(79.9\%) were LIA positive (Figure 1).

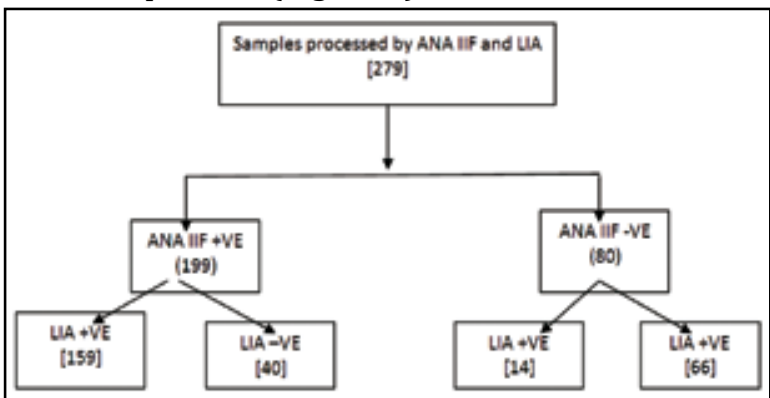

Figure 1: Test results of ANA testing by IIF and LIA method.
The spectrum of various ANA patterns positive by IIF method are shown in Figure 2.

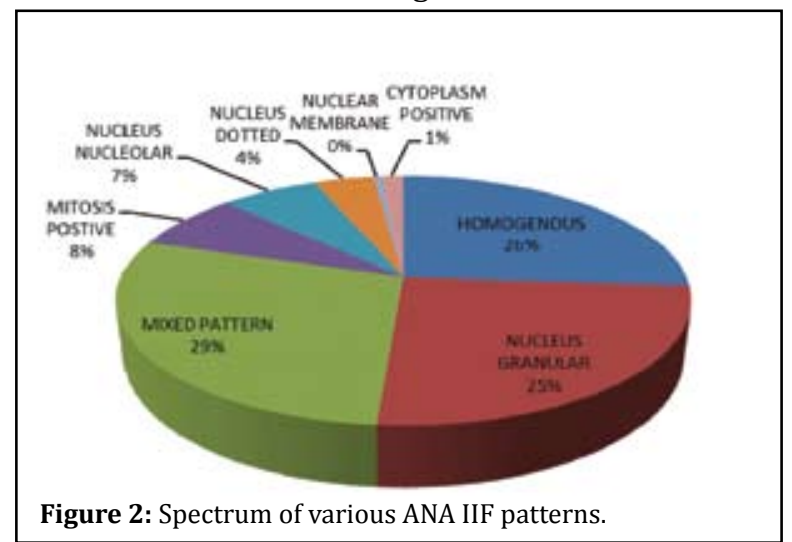


Mixed pattern is the most common ANA pattern, seen in 57(29\%) of the positive 199 ANA IIF samples. In comparison with LIA, the same mixed patterns of autoantigens were observed in 25 (44\%), single autoantigens in 24(42\%) and 8 samples (14\%) were negative. The various autoantigens observed mixed pattern by IIF method are shown in Figure 3. The most common mixed pattern observed was Nucleus homogenous and Nucleus granular seen in $41(72 \%)$ samples.
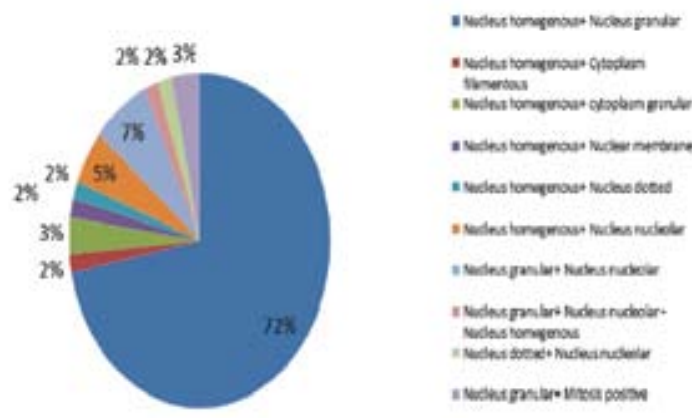

Figure 3: Mixed pattern details by ANA IIF.

The second most common pattern seen in our study are Nucleus homogenous ( $\mathrm{n}=52,26 \%)$.When compared with LIA results, similar autoantigens were observed in $30(58 \%)$, different autoantigens in $8(15 \%), 14(27 \%)$ samples were negative. The various combinations of specific autoantigens observed and the most prevalent among these is shown in Figure 4.

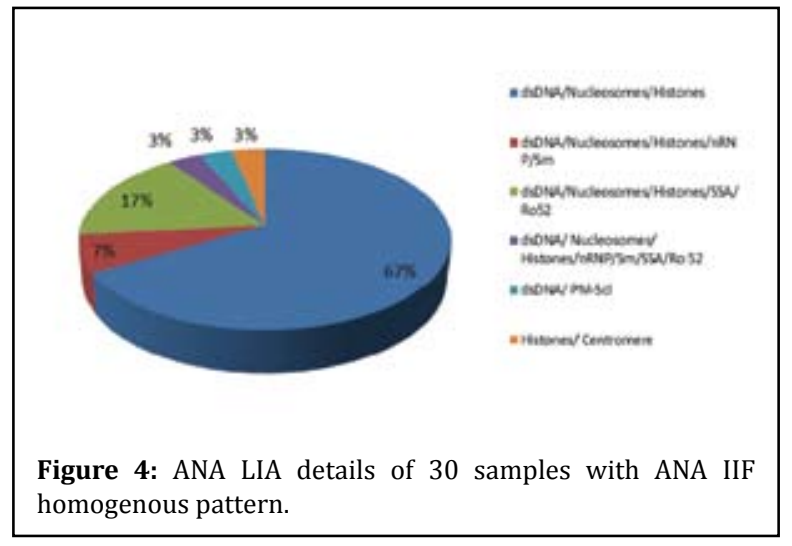

The third most common ANA pattern observed was Nucleus granular $(\mathrm{n}=50,25 \%)$. Correlation with LIA, results showed similarity in $34(68 \%)$ samples and the various combinations of autoantigens seen in LIA are shown in Figure 5.
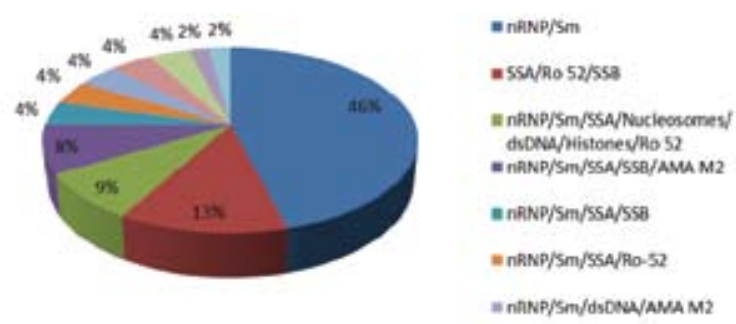

Figure 5: ANA LIA details of 52 samples with ANA IIF granular pattern.

Nucleus nucleolar pattern was observed in 13(7\%) samples. In comparison with LIA, similarity was observed in 6(46\%), different autoantigens in $2(15 \%)$ and $5(39 \%)$ samples were negative probably because LIA test profile does not include fibrillarin, RNA Polymerase and NOR 90 which are observed by ANA IIF. LIA results of 13 Nucleus nucleolar pattern samples are shown in Figure 6.

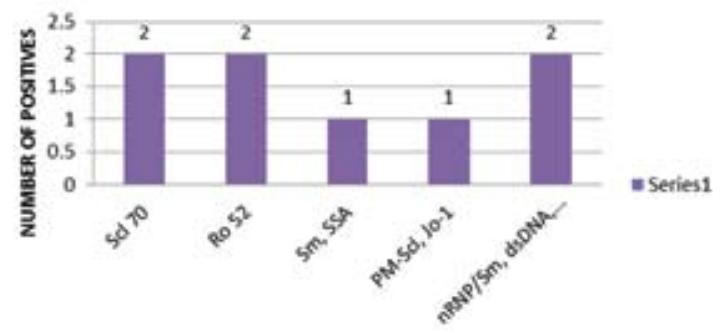

Figure 6: ANA LIA details of 13 samples with ANA IIF nucleus nucleolar pattern.

The other patterns observed in ANA IIF are mitosis positive $(n=15,8 \%)$, nucleus dotted $(n=8,4 \%)$, nuclear membrane $(\mathrm{n}=1,0.35 \%)$ ) and cytoplasm positive( $(n=3,1 \%)$.

In our study, $40(20.1 \%)$ of the ANA IIF positive samples showed negativity with LIA. The ANA pattern observed in these cases were homogenous $13(32.5 \%)$, mixed pattern $8(20 \%)$, mitosis positive $8(20 \%)$, nucleus nucleolar 5(12.5\%), nucleus dotted $3(7.5 \%)$, nucleus granular $2(5 \%)$ and nuclear membrane $1(2.5 \%)$.

ANA IIF negativity was observed in $80(28.6 \%)$ samples. Of these $14(17.5 \%)$ exhibited positivity with LIA. The LIA details of 14 ANA IIF negative 
samples are shown in Figure 7. 66(82.5\%) were negative for ANA by both IIF and LIA methods.

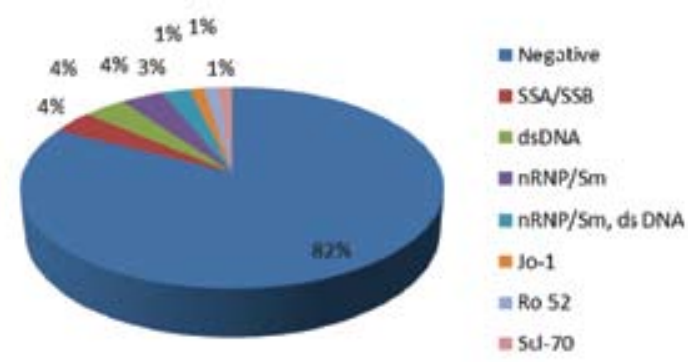

Figure 7: ANA LIA details of 80 samples negative by ANA IIF.

\section{Discussion}

In our study ANA IIF positives were more among the females $(n=171,72.4 \%)$ than males $(n=28,(65.2 \%)$, whereas ANA LIA was positive in $147(62.2 \%)$ and negative in $89(37.8 \%)$ female patient samples. Out of 43 male patient samples, ANA LIA was positive in $23(53.4 \%)$ and negative in $20(34.8 \%)$. This is correlating with the findings of Priyadarshini et al. [7] and Hayashi et al. [8]. In the present study most of the ANA positives were seen in 41-50 years and 31-40 years followed by 21-30 years. Priyadarshini et al. reported most of the ANA positives in the age group 21-30 years followed by 41-50 years [7].

Among the 279 ANA samples processed, 199(71.3\%) samples were ANA IIF positive in 1:100 serum dilutions. Of these positive ANA IIF, 159(79.9\%) were LIA positive and $40(20.1 \%)$ samples were LIA negative. This inconsistency between LIA and IIF is because in LIA according to current reports, only 15 specific antigens can be detected in the clinical laboratory [9] and if the autoimmune disease was caused by antigen, not including the 15 specific antigens, the LIA results were possibly negative $[10,11]$. Based on this, ANA IIF is perceived as the "gold standard" or screening test and LIA as the confirmatory test [13].

ANA IIF negativity was observed in $80(28.6 \%)$ samples. Of these $14(17.5 \%)$ exhibited positivity with LIA. This inconsistency is because for IIF, the low concentration of the autoantigens and the destruction of the antigens during the preparation of the HEp-2 substrate possibly made the result negative [12]. This inconsistency of results between
ANA IIF and LIA was also observed by Sebastian et al. where $17.5 \%$ of ANA IIF positive samples showed negativity with LIA and $13.5 \%$ of ANA IIF negative samples showed positivity by LIA [6].

The various ANA IIF patterns observed in our study are mixed pattern $(29 \%)$, nucleus granular $(25 \%)$, nucleus homogenous (26\%), nucleus nucleolar (7\%), mitosis positive (8\%), nucleus dotted (4\%), nuclear membrane $(0.35 \%)$ and cytoplasmic pattern (1\%). Sebastian et al. have observed homogenous pattern in $45.5 \%$, granular pattern in $35.6 \%$ of IIF positive samples [6]. The mixed pattern in our study is because we have done IIF test only 1:100 dilution and further dilutions of 1:320, 1:1000 and 1:3200 was not done to find out the most prominent pattern in the mixed pattern. Priyadarshini et al. has observed homogenous in $53.8 \%$, granular in $23.07 \%$, nucleolar in $11.53 \%$, cytoplasmic in $3 \%$ of IIF positive samples [7]. Sunitha et al. reported that a cytoplasmic granular pattern in $37 \%$ of the samples and a homogenous pattern in $23 \%$ of the samples [14]. Asli Gamze Sener et al. reported homogenous (54.67\%), granular (18.7\%), centromere $(9.05 \%)$, nucleolar (5.53\%), nuclear dots (4.87\%), cytoplasmic except for golgi and actin (4.07\%), actin $(0.55 \%)$, golgi $(0.21 \%)$, nuclear membrane $(1.21 \%)$ and mixed pattern $(1.14 \%)$ [15].

Homogenous pattern $(n=52,26 \%)$ showed an association with dsDNA, Nucleosomes and histones (67\%) with variable intensities of SSA/Ro-52 (17\%), nRNP/Sm (7\%), PMScl (3\%), centromere (3\%), nRNP/Sm/SSA/Ro 52(3\%). Thus, with homogenous pattern, we can say that the serum had antibodies against dsDNA, nucleosomes and histones in 57.7\% cases which are correlating with Sebastian et al. study of $75 \%$.

Nucleus granular pattern showed a close association with nRNP/Sm, SSA, SSB and Ro52 in $88.4 \%$ of the cases with varying combinations with other patterns showing the predictability of these autoantibodies in samples with granular pattern.

Comparatively centromere B (CB) showed a high specificity (100\%) to the centromere pattern. Mixed pattern sera showed the same mixed patterns of autoantigens in (44\%), single autoantigens (42\%) and negative in $14 \%$.This necessitates the need of testing the sera for further dilutions. As the number of nucleus nucleolar pattern were less, a definite 
correlation was not found between the ANA IIF and the antibodies in LIA.

On the contrary, 14 samples which were LIA positive but ANA IIF negative showed SSA/ SSB/ Ro 52/ nRNP/ Sm in 7 samples, dsDNA in 5 samples, Scl 70 in 2 samples. Similar findings were observed by Sebastian et al. [6], Vos et al. [16] and Hoffman et al. [17]. This explained the fact that LIA was more sensitive than IIF in detecting SSA/ Ro-52/ nRNP/ $\mathrm{Sm}$. This gives a message that LIA testing should be done in suspected cases of autoimmune diseases when ANA IIF is negative. Overall in our study ANA IIF was showing $75.2 \%$ correlation with LIA.

\section{Conclusion}

Antinuclear antibodies (ANA) are the hallmark of autoantibody production in autoimmune disease. ANA IIF test using biochip wells provides a rapid, highly sensitive, most cost effective method for ANA detection. It can be used as a screening test for patients due to its ability to detect multiantigens simultaneously at the same time. Its fluorescent pattern can predict the presence of certain specific antibodies in the sera. LIA can be restricted detection of specific antibodies in all positive ANA IIF samples and also in strongly suspected autoimmune disease patients whose sera showed negative result by IIF method. This could economize on the cost of laboratory investigations for ANA testing in a developing country like India.

\section{Conflict of interest}

The authors declare no conflict of interest.

\section{References}

1. Rabika Dipti T, Shaiful Azam Md, Humayun Sattar Md, Rahman S. Detection of antinuclear antibody in childhood rheumatic diseases by immunofluorescence assay and enzyme immunoassay. Bangladesh Journal of Child Health 2011; 35(2):49-52.

2. Pisetsky DS. Antinuclear antibodies in Rheumatic Disease: A Proposal for a Function based Classification. Scand J Immunol. 2012; 76(3):223-228.

3. Gniewek RA, Stites DP, Thoma MM, Joan FH, Mayumi Nakagawa. Comparison of antinuclear antibody testing methods: immunofluorescence assay versus enzyme immunoassay. Clinical and Diagnostic Laboratory Immunology 1997; 4(2):185-188.

4. Kumar Y, Bhatia A, Ranjana WM. Antinuclear antibodies and their detection methods in diagnosis of connective tissue diseases: A journey revisited. Diagnostic Pathology 2009; $4: 1$.
5. Eric L Greidinger, Robert W Hoffman. Antinuclear antibody testing: Methods, indications and interpretation. Laboratory Medicine 2013; 34(2):113-117.

6. Sebastian W, Roy A, Kini U, Mullick S. Correlation of antinuclear antibody immunofluorescence patterns with immune profile using line immunoassay in Indian scenario. Indian Journal of Pathology and Microbiology 2010; 53(3):427-432.

7. Priyadarshini S, Meenakshisundaram J, Pothini JA. Comparative study of enzyme linked immunosorbent assay (ELISA) with immunofluorescence assay (IFA) for the detection of antinuclear antibodies. International Journal of Pharma and Bio Sciences 2013; 4(4):75-80.

8. Hayashi N, Kawamoto T, Mukai M, Morinobu A, Koshiba M. et al. Detection of antinuclear antibodies by use of an enzyme immunoassay with nuclear HEp-2 cell extract and recombinant antigens: Comparision with immunofluorescence assay in 307 patients. Clinical Chemistry 2001; 47(9):1649-1659.

9. Lee SA, Kahng J, Kim Y, Park YJ, Han K, et al. Comparative study of immunofluorescent antinuclear antibody test and line immunoassay detecting 15 specific autoantibodies in patients with systemic rheumatic disease. J Clin Lab Anal. 2012; 26(4):307-314.

10. Kroshinsky D, Stone JH, Bloch DB, Sepehr A. Case records of the Massachusetts General Hospital. Case 5-2009. A 47year-old woman with a rash and numbness and pain in the legs. N Engl J Med. 2009; 360(7):711-720.

11. Hansson- Hamlin H, Ronnelid J. Detection of antinuclear antibodies by the Inno-Lia ANA update test in canine systemic rheumatic disease. Vet Clin Pathol 2010; 39(2):215-220.

12. TanakaN, Muro Y. Anti-SSA/Ro antibody determination by indirect immunofluorescence and comparison of different methods of antinuclear antibody screening. Mod Rheumtol. 2008; 18(6):585-592.

13. Meroni PL, Schur PH. ANA screening :an old test with new recommendations. Ann Rheum Dis. 2010; 69(8):14201422.

14. Sunitha KG, Andan ST, Bharani K, Seena Raghunathan, Soji Cyriac, et al. Screening of autoantibodies using indirect immunofluorescence in autoimmune predisposed individuals Discovery 2012; 1(1):8-13.

15. Sener AG, Afsar I, Demirci M. Evaluation of antinuclear antibodies by indirect immunofluorescence and line immunoassay methods: four years data from Turkey. APMIS 2014.

16. Vos PA, Bast EJ, Derksen RH. Cost effective detection of non anti-dounle stranded DNA antinuclear antibody specificities in daily clinical practice. Rheumatology (Oxford) 2006; 45:629-635.

17. Hoffman IE, Peene I, Veys EM, De Keyser F. Detection of specific antinuclear reactivities in patients with negative anti-nuclear antibody immunofluorescence screening tests. Clin Chem 2002; 48:2171-2176. 